\title{
La cultura en Las organizaciones
}

Virginia Isabel Camacho Álvarez *

\section{Resumen}

Así como los individuos cuentan con una personalidad propia que está formada por una serie de características relativamente permanentes y estables, así las organizaciones tienen una personalidad que llamamos cultura. El aspecto que se comparte de la cultura es aquel que perciben los empleados no importa en realidad su nivel jerárquico, se basa en las percepciones y tienden a describir a la cultura en términos muy similares, pueden ser denominados artefactos visibles según la clasificación que hace Schein (Stonner, 1996:200). Por otro lado el grado de acuerdo o desacuerdo entre los miembros de una organización determina su fortaleza haciendo que se tornen en adaptables o no adaptables, concluyendo en que una cultura no adaptable puede ser más peligrosa que una débil.

\section{Abstract}

Organizations, like individuals, have their own personality formed by a group of relatively permanent and stable characteristics which is called culture. The shared aspect of culture as perceived by employees (regardless of rank) is based upon perceptions and tends to describe the culture in similar terms; according to Schein's classification, they can be called visible artefacts.

On the other hand, the level of agreement or disagreement amongst members of an organization determines its strength becoming adaptable or non-adaptable with the understanding that a non-adaptable culture can be more dangerous than a weak one.

La cultura en las organizaciones, entendiendo el término organización como la "asociación de personas regulada por un conjunto de normas en función de determinados fines", no surge de manera espontánea, sus costumbres actuales, tradiciones y la forma general de hacer las cosas en ellas tienen en gran parte su origen en lo que se ha hecho antes, de tal manera que el grado de éxito que se tiene hoy con los esfuerzos y experiencias anteriores, nos lleva a la fuente primaria de la cultura de una organización: sus fundadores.

La palabra cultura proviene del latín cults (cultivado) y ura (acción, resultado de una acción) y significa cultivo, agricultura, instrucción. La cultura a través del tiempo ha sido una mezcla de rasgos, distintivos espirituales y afectivos, que caracterizan a una sociedad o grupo social en un período determinado. 
Comprende además formas de vida, ritos, artes, inventos, tecnología, costumbres valorales, derechos humanos fundamentales, tradiciones y creencias.

* Maestría en Administración. Directora de Extensión Universitaria en la Universidad La Salle Pachuca

vcamacho@lasallep.edu.mx

Como señala Zubiría Samper (1998:21) con respecto a la cultura en la época moderna:

...nos independiza de la naturaleza animal para configurar lo verdaderamete humano. Esto es, que gracias a ella superamos el animalitas para llegar al humanitas, por tanto podemos decir que la cultura es también el cultivo de la espiritualidad humana y se convierte en el camino hacia la humanización.

Abravanel (1992:202) menciona que la cultura también es resultado del aprendizaje humano, es la manera que tiene la gente de potenciar sus experiencias concretas en un mundo fenomenal o conceptual.

Ahora bien la cultura en las organizaciones puede percibirse en lo que la gente piensa, hace y siente; implica la adquisición y transmisión de estos patrones de conducta en el transcurso del tiempo, lo que significa que la cultura de una organización tiende a ser estable y no puede ser cambiada aceleradamente.

Cada organización posee una cultura que le es propia: un sistema de creencias y valores compartidos al que se apega al elemento humano que las conforma. La cultura corporativa crea, y a su vez es creada, por la disposición del medio ambiente interno; en consecuencia, condiciona el grado de cooperación y de dedicación, y el arraigo de los propósitos institucionales dentro de una organización. En este sentido la principal responsabilidad de quien dirige consiste en fijar el modo, el ritmo y la importancia que se dé a los planes estratégicos de los cuales son responsables.

Kast y Rosenzweig (1988:701-704) sostienen que la cultura es lo que mantiene social o normativamente unida a una organización. Expresa los valores o ideales sociales o creencias que los miembros de la organización llegan a compartir, manifestados en elementos simbólicos, como mitos, rituales, historias, leyendas y un lenguaje especializado. La cultura podría ser definida como el complemento de intercambio de las características comunes que influyen en la respuesta de un grupo humano a su medio.

Las organizaciones son en sí mismas sujetos productores de cultura, son vistas como instrumentos sociales que producen bienes y servicios, y también producen 
rituales, leyendas y ceremonias como formas culturales distintivas. Aunque las organizaciones en sí están sumergidas en un contexto cultural más amplio, el interés primordial está en las actividades socioculturales que se desarrollan dentro de las organizaciones.

La cultura organizacional incluye lineamientos perdurables que dan forma al comportamiento. Cumple con varias funciones importantes al transmitir un sentimiento de identidad a los miembros de la organización, al facilitar el compromiso con algo mayor que el yo mismo, al reforzar la estabilidad del sistema social y al ofrecer premisas reconocidas y aceptadas para la toma de decisiones.

En general la explicación más simple que se hace acerca del término cultura organizacional, se refiere a un sistema de valores compartidos que principalmente nos dicen qué ven los empleados y cómo responden al enfrentar un problema, sin embargo también el tipo de cultura

organizacional en muchos casos puede llegar a reprimir las sugerencias de los empleados para conceptualizar, definir, analizar y resolver el problema de la forma más efectiva.

Horacio Andrade (Trelles:161-164) considera al término de cultura organizacional con una visión diferente e introduce el término sistema cultural definiéndolo como "aquel que se integra por el conjunto de valores y creencias que comparten las personas que pertenecen a él, y por las múltiples formas en que se manifiestan dichos valores y creencias". Dicho modelo permitiría considerar sistemas culturales a una mayor variedad de agrupaciones humanas. La organización constituiría entonces, un sistema cultural que constaría de tres elementos primordiales: valores, creencias y manifestaciones, éstas últimas aplicadas a la organización, las clasifica en:

- Conceptuales-simbólicas: incluyen la filosofía de la organización (misión, objetivos,

prioridades, programas y estrategias básicas), recursos simbólicos y mitología.

- Conductuales: a ellas corresponden el lenguaje, el comportamiento no verbal, el ri-

tual y las distintas formas de interacción en la organización.

- Estructurales: las integran las políticas, las normas, los procedimientos, el sistema

de status interno, el liderazgo formal e informal visto como estructura de poder. 
- Materiales: son los recursos materiales de la organización: la tecnología, instalacio-

nes, mobiliario y equipos.

El mismo autor señala que existe una interdependencia constante entre la cultura y sus manifestaciones, ya que estas reflejan la cultura imperante, al tiempo que la enriquecen. Cada organización valorará y enfatizará distintivamente los elementos culturales, hecho que, indudablemente, define y hace ecos de su cultura.

Robbins (1996:685) al definir a la cultura organizacional dice que implica varias cosas, en primera instancia es una percepción; los individuos perciben la cultura de la organización, basándose en lo que ven o escuchan dentro de la empresa, y, no obstante que los empleados tengan antecedentes distintos o trabajen en un nivel distinto dentro de la compañía, tienden a describir la cultura de la misma en términos similares, es lo que se considera el aspecto compartido de la cultura. En segunda instancia "la cultura organizacional" es un término descriptivo; se ocupa de cómo perciben los miembros a la organización, no de si les gusta o no; luego, es descriptiva más que evaluativa.

La definición más aceptada de Cultura Organizacional es la de Edgar $\mathrm{H}$. Shein, quiendice que es el patrón de premisas básicas que un determinado grupo inventó, descubrió o desarrolló en el proceso de aprender a resolver sus problemas de adaptación externa y de integración interna y que funcionaron suficientemente bien a punto de ser consideradas válidas $y$, por ende, de ser enseñadas a nuevos miembros del grupo como la manera correcta de percibir, pensar y sentir en relación a estos problemas".

Schein, incluso, propone que la comprensión de la cultura organizacional sea parte integrante del propio proceso administrativo. Un estudio de cultura que se haga dentro de las organizaciones permite entender las relaciones de poder, las reglas no escritas, lo que es tenido

como verdad, etc. En este sentido aclara una serie de comportamientos considerados aparentemente evidentes, permitiendo planear el trabajo de forma coherente con la realidad de organización.

Señala también, que los paradigmas de la cultura de una empresa poseen siempre un eslabón con la cultura del entorno en el que está inserta la empresa. "En este sentido, los paradigmas de la cultura organizacional son versiones adaptadas de paradigmas culturales más amplios". Por tanto, esto reafirma que un extranjero no podría realizar un estudio de cultura por no contar con las 
experiencias y conocimientos para hacer las conexiones debidas con la cultura del país.

Schein sugiere que la cultura existe en una organización a partir de la observación de tres niveles: El primer nivel, es el de los artefactos visibles, que comprende el entorno físico de organización, su arquitectura, los muebles, los equipos, el vestuario de sus integrantes, el patrón de comportamiento visible, documentos, cartas, etc. Son datos bastante fáciles de conseguir pero difíciles de interpretarse. El análisis de este nivel puede ser engañoso, ya que, muchas veces, no se consigue comprender la lógica que está por detrás de estos datos. En este nivel podemos observar la manifestación de cultura pero nunca podremos saber su esencia.

El segundo nivel, es el de los valores que dirigen el comportamiento de los miembros de la empresa. Su identificación, solamente es posible a través de entrevistas con los miembros clave de la organización.

Finalmente, el tercer nivel es el de los supuestos inconscientes, que revelan más confiadamente la forma como un grupo percibe, piensa, siente y actúa. Estos supuestos son construidos a medida que se soluciona un problema eficazmente. Con el pasar del tiempo estas premisas dejaron de ser cuestionadas, constituyéndose "verdades" y volviéndose inconscientes. Este último nivel está compuesto por las siguientes dimensiones:

Dimensión 1. Relación de la organización con el entorno externo

Dimensión 2. Naturaleza, de la verdad y de la realidad.

Tradición; Religión/dogma/moral; Revelación a través de una autoridad; Racional; Resolución de conflictos a través de debates seguidos; Ensayo-error; Experimento Científico.

Dimensión 2a. Naturaleza del tiempo

Dimensión 2b. Naturaleza del espacio

Dimensión 3. El contexto de la naturaleza humana

Dimensión 4. La naturaleza de la actividad humana

Stoner (1997, Pág. 200) dice que aunque muchos aspectos de la cultura organizacional son muy evidentes, muchos otros son menos visibles, esto hace que se parezca a un iceberg. En la superficie están los aspectos evidentes o abiertos como las metas de la organización expresadas de manera formal, la tecnología, estructura, políticas y procedimientos, así como los recursos 
financieros. Bajo la superficie están los aspectos ocultos o encubiertos, los aspectos informales en la vida de la organización; estos incluyen percepciones, actitudes y sentimientos compartidos sobre la naturaleza humana y sus relaciones, y lo que puede recordar o recordará la organización.

Stoner (1996, pág. 202) menciona que en un estudio hecho por investigadores de la Escuela de Administración de Harvard, donde trataron de definir cuáles factores hacen que las culturas organizacionales tengan más éxito que otras. Lo que intentaron encontrar fueron los factores de éxito, para que otras organizaciones pudieran emprender programas que les ayudaran a cambiar sus culturas a fin de hacerlas más exitosas.

El resultado del estudio llegó a cuatro conclusiones principales:

1. La cultura corporativa puede tener un impacto significativo en el desempeño eco-

nómico de la empresa, a largo plazo.

2. La cultura corporativa, quizá, será un factor aún más importante que determine el

éxito o fracaso de las empresas en la próxima década.

3. No son raras las culturas corporativas que inhiben el desempeño financiero sólido,

a largo plazo; éstas se desarrollan con gran facilidad, incluso en empresas que están

llenas de personas razonables e inteligentes.

4. Sí se puede lograr que las culturas corporativas, aunque sean difíciles de cambiar,

refuercen más el desempeño.

Para identificar e interpretar el contenido de la cultura Daft $(2000,371)$ dice que se requiere que la gente haga deducciones con base en artefactos observables. Los artefactos se pueden estudiar pero es difícil descifrarlos con precisión. Algunos de los aspectos observables, típicos e importantes de la cultura son, las ceremonias y los ritos, las anécdotas, los símbolos y el lenguaje.

Fortaleza y adaptación de la Cultura 
Daft también menciona que la fortaleza de una cultura se refiere al grado de acuerdo entre los miembros de una organización sobre la importancia de valores específicos. Afirma que si existe un convenio, un consenso amplio sobre la importancia de tales valores, la cultura es identificada y fuerte; si existe poco acuerdo es débil.

Con respecto a la adaptación, las culturas corporativas adaptables a diferencia de las no adaptables tienen valores y patrones de comportamiento diferentes. En las adaptables los directivos se preocupan por los clientes o usuarios y los empleados, y valoran los procesos que favorecen a un cambio útil. La conducta es tolerante, los directivos inician el cambio cuando se estima necesario, inclusive si está acompañado de cierto riesgo. Estas culturas, fuertes y saludables ayudan a las organizaciones a adaptarse al entorno externo.

A diferencia de las no adaptables en las que los directivos están más preocupados por ellos o por un proyecto individual. Sus actitudes desalientan la toma de decisiones con riesgo y no favorecen el cambio. Las culturas fuertes y no saludables pueden estimular a una organización a marchar resueltamente en una dirección equivocada. Una compañía fuerte no siempre asegura el éxito, a menos que se estimule una adaptación saludable al entorno externo. La conclusión a la que se llega es que una cultura fuerte que no estimula la adaptación puede ser más dañina que una cultura débil.

A lo largo de este trabajo se ha podido observar que los valores forman parte importante de la cultura y que cuando ésta es fuerte y adaptable lleva a las organizaciones a cumplir con su

misión más fácilmente, por lo que las organizaciones que quieren alcanzar sus metas deben evitar que los valores sean vistos como productores de utilidades o ahorradores de dinero, sino considerarlos porque deben formar parte de la conciencia de todos los que trabajan para la organización y que den por resultado una verdadera transformación social.

Hay factores que se unen para minimizar la praxis de la ética en las organizaciones, los cuales deben tomarse muy presentes si deseamos inculturar la práctica de los valores en las mismas, el primero es que la falta de salud financiera aletarga las conciencias, por lo que si tenemos una organización sana es más fácil hacer que se ejerciten; el siguiente, es que cuando hablemos de modernizar a nuestras organizaciones que no sólo sea en el ámbito de la tecnología, sino que se fortalezcan sus valores éticos y se afiance su cultura; y por último combatir arduamente el hábito prevalecido de la corrupción. 
La conclusión más importante a la que se llega es que las organizaciones, públicas o privadas, consideren en sus programas de formación y actualización continua para el personal, que no sólo se dé en el ámbito de las competencias profesionales o laborales sino que se haga un énfasis especial en la formación humana.

\section{FUENTES DE CONSULTA}

Abravanel, Allaire, Firsirotu, Hobbs, Poupart y Simard. Cultura Organizacional. 1992 .Aspectos Teóricos, Prácticos y Metodológicos. Santa Fe de Bogotá, Colombia: Fondo Editorial Legis.

DaftRichard L. , Teoría y Diseño Organizacional. México. 1998 Internacional Thomson Editores. ISBN 968-7529-78-4

KastFremont E. y Rosenzweig James E. , Administración en las organizaciones, Enfoque de sistemas y de contingencias. México 1988. Editorial McGraw Hill. ISBN 968-451-449-2.

RobbinsStephen P. , Comportamiento Organizacional, Teoría y Práctica. México 1996. Editorial Prentice Hall.

SchwanitzDietrich. , La Cultura Todo lo que hay que saber. Décima edición. España 1999. Editorial Taurus.

StonerJames A. F., Freeman R. Edward y Gilbert Daniel R. Jr. (1996) Administración. Sexta edición. México 1996. Editorial Prentice Hall. ISBN 968-880685-4.

ZubiríaSamper Sergio de, Trujillo Ignacio Abello y Tabares Marta, (1998), Conceptos básicos de administración y gestión cultural. Segunda edición. Madrid, España 1998. Editorial de la OEI, ISBN 84-7666-121-5.

\section{ELECTRÓNICAS}

ScheinEdgar H. Liderazgo y Cultura Organizacional. $2^{\text {a }}$ Edición http://www.solonline.org/reflections/authors/author?author_id=107657. Página consultada 23 de julio de 2007.

TRELLES, Rodríguez I. Comunicación y cultura organizacional: entramados e interrelaciones. Espacios 2001; (5):25-8

http://www.gerencie.com/cultura-organizacional.html 